\title{
Unusual geodes ('bone cysts') in rheumatoid arthritis
}

\author{
M. I. V. JAYSON*†, A. ST. J. DIXON*. AND P. YEOMAN*‡
}

From the ${ }^{*}$ Royal National Hospital for Rheumatic Diseases, Bath, and $\dagger$ Department of Medicine, University of Bristol, and the $\ddagger$ Bath and Wessex Orthopaedic Hospital

The geologists' term 'geode' is used in continental Europe to describe the radiological appearance of cavities in the bone ends in patients with various forms of arthritis (Jayson, Rubenstein, and Dixon, 1970). We describe here a patient with rheumatoid arthritis and unusually large geodes, the contents of which were calcified-an appearance which, we believe, has not previously been described.

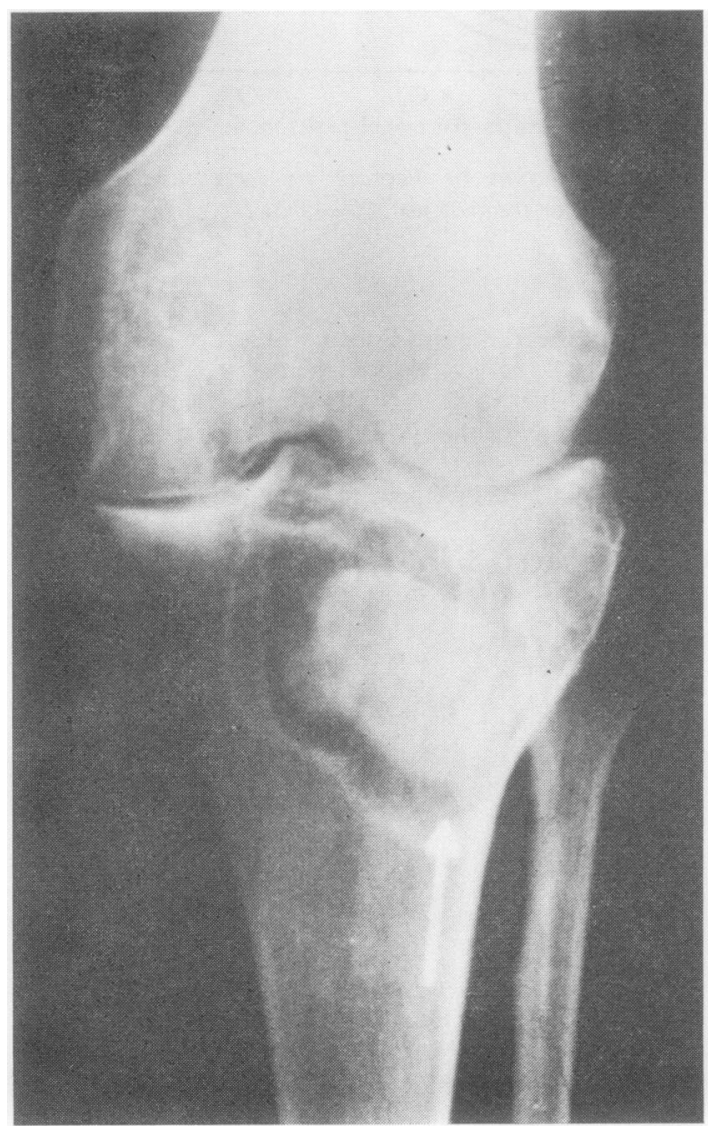

\section{Clinical findings}

A 60-year old male clerk had suffered from classical nodular seropositive rheumatoid arthritis for 20 years. $\mathrm{He}$ had been treated with tetracosactrin for 2 years and had been working until 18 months before admission to hospital. He had been unable to walk for the previous 5 months. The knee joints were the most severely affected.

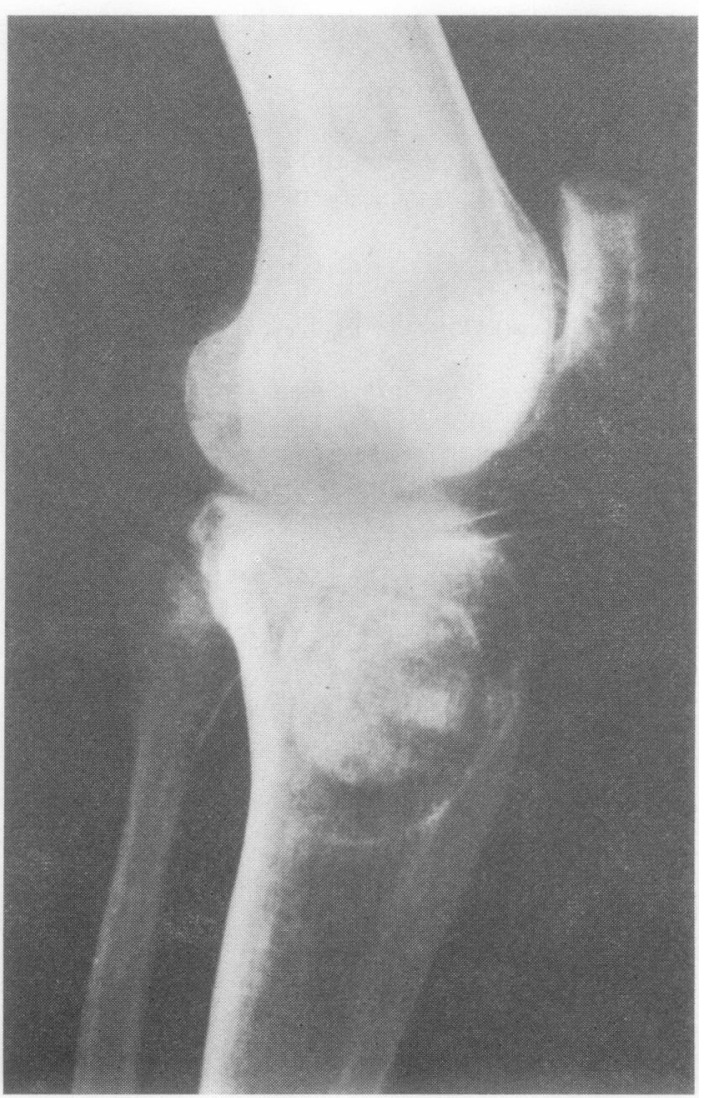

FIG. $1 a, b$ Left knee. Antero-posterior and lateral views 
LEFT KNEE

This flexed with much crepitus between $15^{\circ}$ and $150^{\circ}$. There was a small effusion and some synovial thickening. $X$ rays (Fig. $1 a, b)$ revealed marked loss of articular cartilage in the tibiofemoral compartments, particularly on the lateral side. A large cavity or geode occupied almost the whole of the upper end of the tibia and contained a well-defined dense radio-opaque mass. There appeared to be a connection between the upper border of the geode and the area between the two tibial plateaux. Tomography confirmed these findings and also revealed large geodes in the lower end of the femur. Arthrography was performed by the method of Jayson and Dixon (1970). No filling of the tibial geode was obtained, but there was a small popliteal cyst.

Under local anaesthesia the pressure within the tibial geode was measured by the method of Jayson and others (1970). The intrageodal pressure was the same as the atmospheric pressure and did not vary with quadriceps contraction, flexing the joint, or external pressure on the knee. $10 \mathrm{ml} .60$ per cent. megulamine iothalamate (Conray 280) was injected directly into the geode and $X$ rays were obtained before and after exercise. Even 30 minutes after injection the dye failed to pass from the geode into the joint space but instead remained localized within the cavity (Fig. 2).

\section{Surgery}

A McKee knee arthroplasty was undertaken. At operation the joint capsule was found to be very tight with marked chronic synovial hypertrophy. The articular cartilage showed ulceration in some areas and marked thinning in others. On transection of the tibia, the large geode was entered. It was filled with gelatinous creamy material and there was a friable calcareous mass in the centre, corresponding to the radio-opaque lesion (Fig. 3 ). The geode

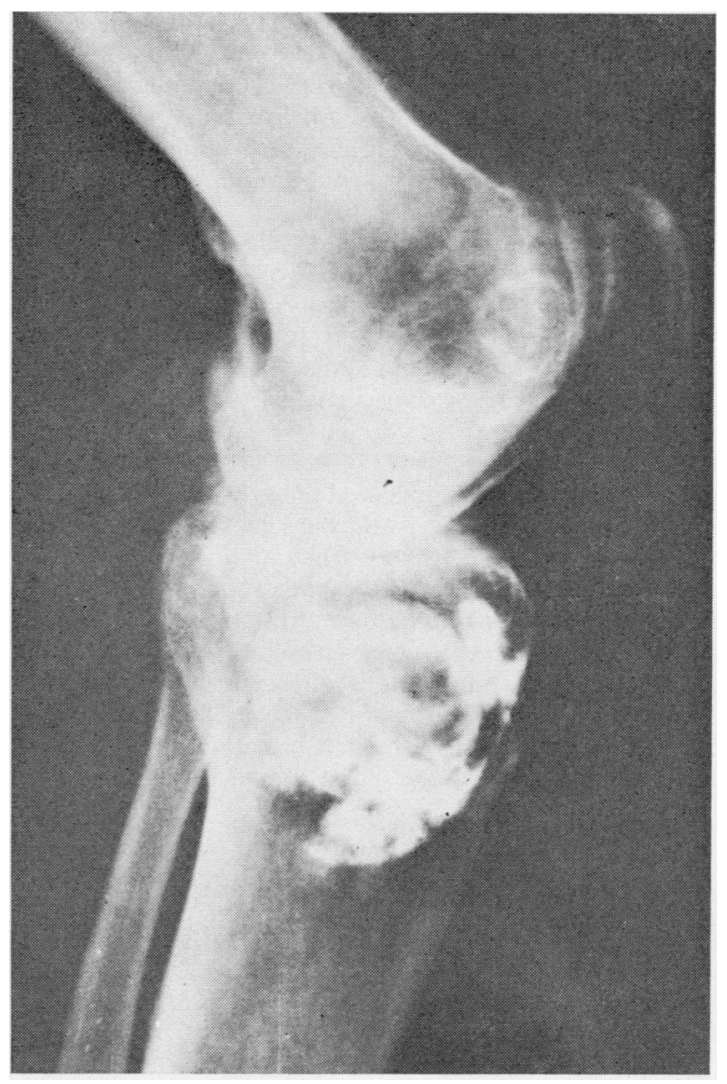

FIG. 2 Left knee. Cystogram

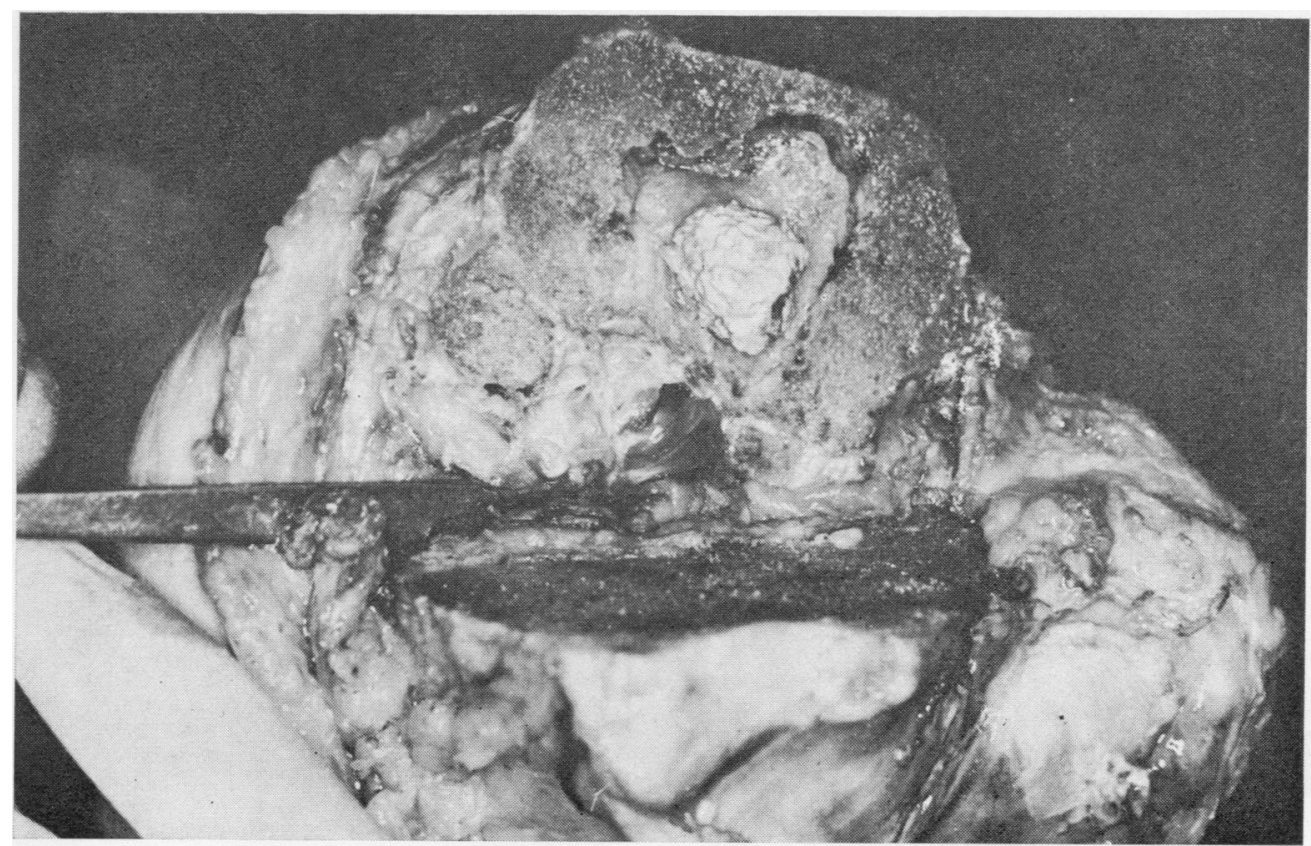

FIG. 3 Left knee. Tibial geode containing calcareous mass 
was lined by a membrane and then by hard bony walls. The upper end of the cavity removed with the tibial plateaux was obstructed by dense fibrous material. With considerable difficulty it was possible to demonstrate a very narrow channel from the apex of the cavity communicating with the joint between the origins of the anterior and posterior cruciate ligaments.

On transection, another large geode was demonstrated in the medial femoral condyle. This was filled with pale gelatinous material. On examination with a curved narrow probe, a tortuous channel was found leading into the intercondylar notch of the knee joint just behind an overlapping lip of articular cartilage.

\section{RIGHT KNEE}

This was more severely involved than the left. There was a $30^{\circ}$ fixed flexed deformity and the knee flexed with much crepitus to $150^{\circ}$. There was synovial thickening but no effusion. A lateral view of the knee suggested that there was some posterior subluxation of the tibia. $X$ ray of this knee (Fig. $4 a, b$ ) revealed an even larger geode in the upper end of the tibia but without any central radio-opaque mass. Tomography demonstrated the geode in more detail but also confirmed that the geode in the lateral femoral condyle contained a dense lesion similar to that in the left

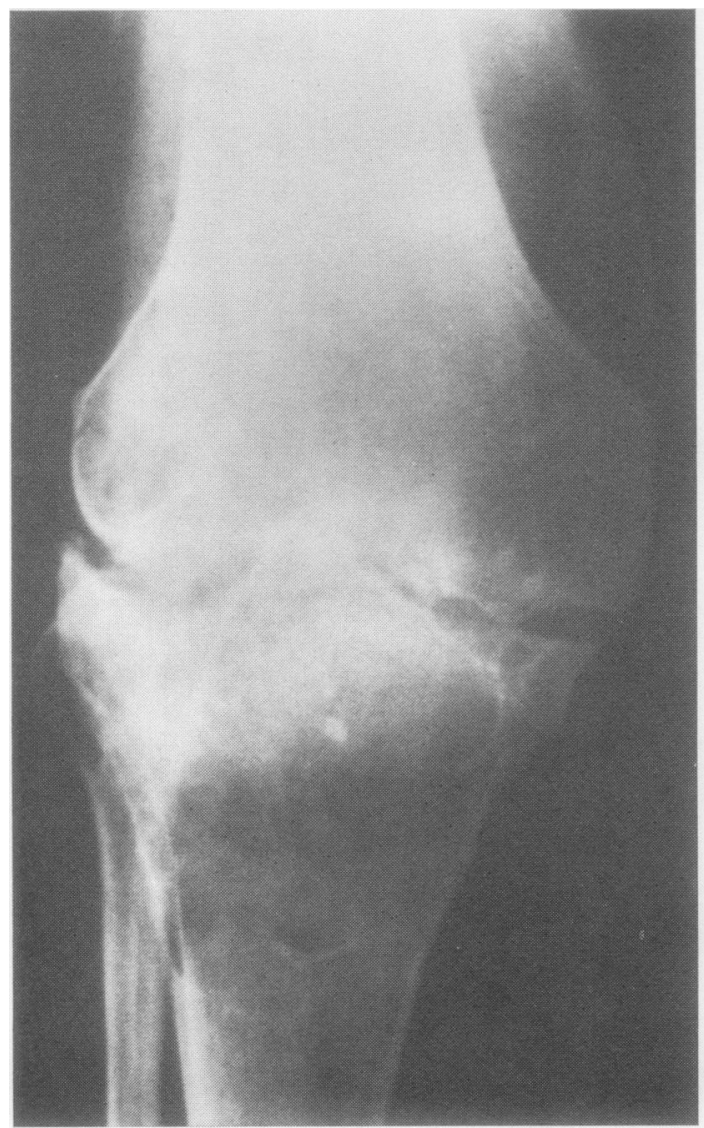

tibia. On arthrography, the dye failed to pass into any of the geodes but did fill a large posterior popliteal cyst. The pressures within the right tibial geode were measured and varied widely between -10 and $+30 \mathrm{~mm}$. $\mathrm{Hg}$. On cystography on this occasion, the dye passed from within the tibial geode into the joint space and the popliteal cyst (Fig. 5).

\section{Surgery}

A McKee knee arthroplasty was performed as before. There was a very tight joint capsule with synovial hypertrophy and gross damage to the articular surfaces. On transection of the tibia, a very large cystic space filled with gelatinous material was found. A large geode with a central friable white calcareous mass was also present in the lateral femoral condyle.

Postoperative convalescence was uneventful and the patient is beginning to walk with the aid of appliances.

\section{HISTOLOGY}

The synovium showed the typical changes of chronic rheumatoid arthritis. The geodes were lined by hyaline fibrous tissue and contained necrotic debris some of which was calcified.

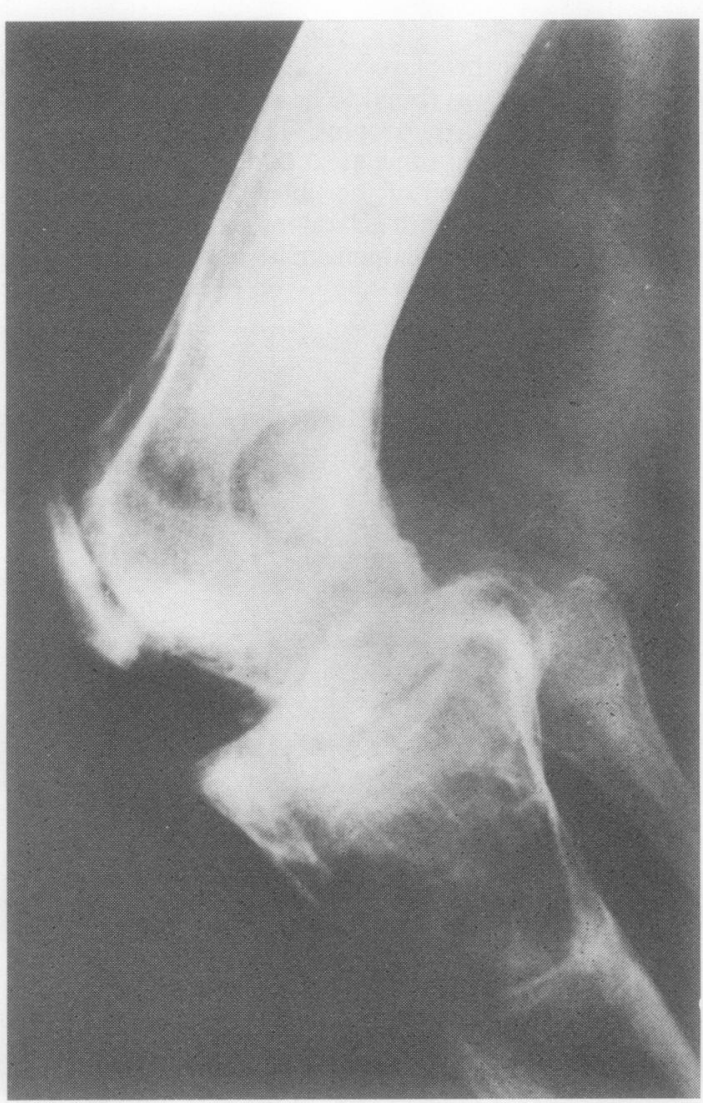

The geode in the lateral femoral condyl contains a

FIG. $4 a, b$ Right knee. Antero-posterior and lateral views. central radio-opaque mass 


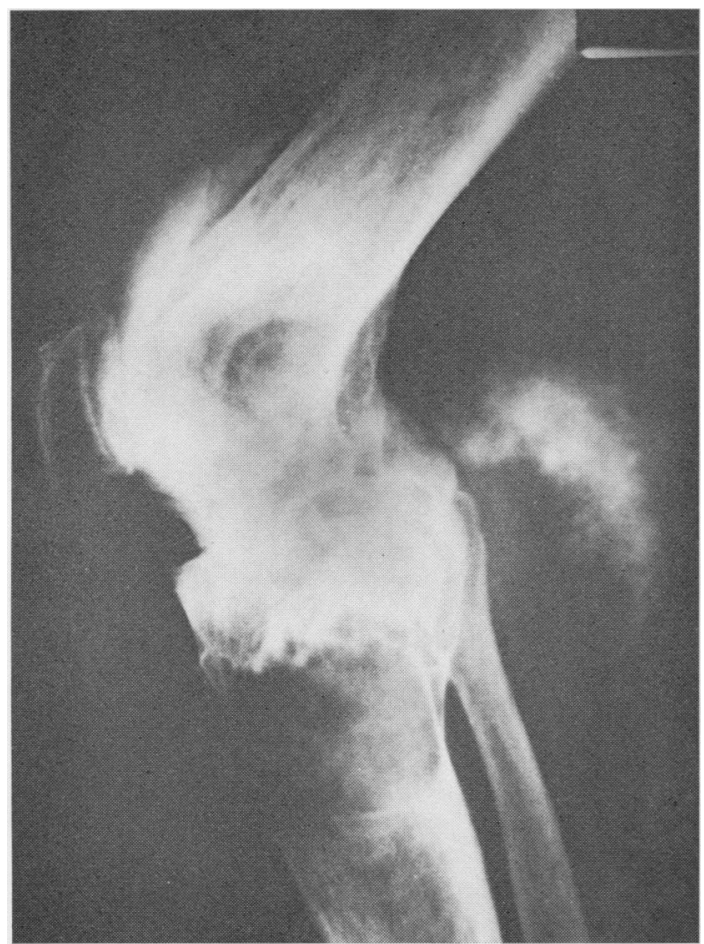

FIG. 5 Right knee. Cystogram

\section{Discussion}

Geodes are commonly found in association with rheumatoid arthritis of the knee. Fletcher and Rowley (1962) and Soila (1963) thought that they were due to primary defects within the bone ends and that they did not connect with the joint surfaces. However, Freund (1940), Cruickshank, Macleod, and Shearer (1954), and Bywaters (1964) produced histological evidence that they did communicate, and Kersley, Ross, Fowles, and Johnson (1954) were able to demonstrate this by macrotomography. Castillo, El Sallab, and Scott (1965) thought that the size and extent of the geode formation in affected hands were directly related to the degree of manual work performed by the patient. Jayson and others (1970) were able to show that alterations in the intra-articular pressure could be transmitted directly into the geode. They showed that use of a swollen joint produces a high intra-articular pressure which is not transmitted across the articular cortex in a normal joint but which could, in patients whose articular surfaces have been eroded and weakened by disease, lead to extrusion of the joint contents into the bone ends.

In our patient the geodes were considerably larger than any that we have previously seen. We have, however, been able to demonstrate a direct connection between the geodes and the joint space. Debris accumulates within the rheumatoid geodes so that it is difficult for radio-opaque dye introduced into the joint to pass into the geode. However, dye was able to pass from the right tibial geode directly into the joint space. The geode in the left tibia presented a unique appearance which we have not previously seen and which we have been unable to find described elsewhere. Dye failed to pass from within its cavity into the joint space, and at subsequent dissection the connection between the geode and the space was closed by dense fibrous tissue. We believe that this geode was originally formed by the same mechanism as the others. Perhaps when the patient ceased walking high pressures were no longer generated within the knee. The communication between the knee and geode then closed and was obliterated by the dense fibrous tissue. As the geode contents remained static, calcium aggregated within its centre, producing the lesion described. Similar changes were present in the smaller geode in the right femoral condyle.

\section{Summary}

A patient is described with large geodes around the knee joints. Geodes in the upper end of the left tibia and in the right lateral femoral condyle each presented a unique feature-a large central radio-opaque mass. The radiological, tomographic, arthrographic, and cystographic features are presented together with the findings on measurement of pressures within the geodes and at operation. The central masses probably developed as a consequence of closure of the connection between the joint space and the geodes.

This study was performed with the aid of grants from the Arthritis and Rheumatism Council and the Association of Friends of the Royal National Hospital for Rheumatic Diseases. M.I.V.J. is in receipt of a research grant from the Medical Research Council.

\section{References}

Bywaters, E. G. L. (1964) 'The hand' in 'Radiological Aspects of Rheumatoid Arthritis'. Excerpta Medica, International Congress Ser. No. 61, p. 43

Castillo, B. A., El Sallab, R. A., AND Scott, J. T. (1965) Ann. rheum. Dis., 24, 522 (Physical activity, cystic erosions, and osteoporosis in rheumatoid arthritis)

Cruickshank, B., Macleod, J. G., and Shearer, W. S. (1954). J. Fac. Radiol. (Lond.), 5, 218 (Subarticular pseudocysts in rheumatoid arthritis)

Fletcher, D. E., AND Rowley, K. A. (1952) Brit.J. Radiol., 25, 282 (The radiological features of rheumatoid arthritis) 
FREUND, E. (1940) Edin. med. J., 47, 192 (The pathological significance of intra-articular pressure)

JAYSON, M. I. V., AND DIXON, A. ST. J. (1970) Ann. rheum. Dis., 29, 415 (Valvular mechanisms in juxta-articular cysts)

, RuBensteIn, D., AND Dixon, A. St. J. (1970) Ibid., 29, 496 (Intra-articular pressure and rheumatoid geodes (bone 'cysts'))

KeRSLEY, G. D., Ross, F. G. M., Fowles, S. J., AND Johnson, C. (1964) Ibid., 23, 280 (Tomography in arthritis of the small joints)

SollA, P. (1963) Acta rheum. scand., 9, 231 (The causal relations of rheumatoid disintegration of juxta-articular bone trabeculae) 